\title{
ESTUDO DE PRÉ-FORMULAÇÃO DE SHAMPOO PARA TRATAMENTO DA ALOPECIA ANDROGENÉTICA CONTENDO CAFEÍNA COMO ATIVO
}

DOI: $10.22289 / 2446-922 X . V 4 N 1 A 9$

Lilian de Abreu Ferreira ${ }^{1}$

Andrielle Sousa Amaral

\section{RESUMO}

A alopecia androgenética se caracteriza pela perda progressiva dos cabelos. Para o tratamento tópico dessa condição, a via folicular é considerada a mais rápida na disponibilização dos ativos. Recentemente, alguns estudos mostraram que a cafeína possui efeitos benéficos em pacientes acometidos por esta condição. $O$ objetivo foi realizar um estudo de pré-formulação de shampoo contendo cafeína para tratamento da alopecia androgenética. Foi realizada a manipulação da base em shampoo para a veiculação do ativo cafeína, e sua estabilidade foi analisada através de parâmetros físico-químicos prédeterminados. As amostras foram mantidas nas temperaturas $5 \pm 2^{\circ} \mathrm{C}, 40 \pm 2^{\circ} \mathrm{C}$ e $20 \pm 2^{\circ} \mathrm{C}$ por 15 dias. A formulação apresentou boa aparência e homogeneidade nos tempos T0, T7 e T15, viscosidade de $11386 \mathrm{mPa}$.s, $\mathrm{pH}$ 6,28 e densidade $1,024 \mathrm{~g} / \mathrm{mL}$, mantendo-se estável até a última análise, sendo a formulação proposta viável ao universo magistral.

Palavras-chave: Alopecia; Cafeína; Estabilidade de cosméticos.

\section{ABSTRACT}

Androgenetic alopecia is characterized by progressive loss of hair. For topical treatment, the follicular route is considered the fastest for the availability of the assets. Recently, some studies have shown that caffeine has beneficial effects in patients with this condition. The objective was to carry out a preformulation study of caffeine-containing shampoo for the treatment of androgenetic alopecia.The manipulation of the base in shampoo was performed for the placement of caffeine, and its stability was analyzed through pre-determined physical-chemical parameters. The samples were maintained at temperatures $5 \pm 2^{\circ} \mathrm{C}, 40 \pm 2^{\circ} \mathrm{C}$ and $20 \pm 2^{\circ} \mathrm{C}$ for 15 days. The formulation presented good appearance and homogeneity at times T0, T7 and T15, viscosity of $11386 \mathrm{mPa} . \mathrm{s}, \mathrm{pH} 6.28$ and density $1,024 \mathrm{~g} / \mathrm{mL}$, remaining stable until the last analysis, being the proposed formulation viable to the masterful universe.

Keywords: Alopecia; Caffeine; Cosmetic Stability.

\footnotetext{
${ }^{1}$ Endereço eletrônico de contato: lyabreu@hotmail.com

Recebido em 22/10/2017. Aprovado pelo conselho editorial para publicação em 24/10/2017.
}

Rev. Psicol Saúde e Debate. Fev., 2018:4(1): 147-160. 


\section{INTRODUÇÃO}

A alopecia androgenética (AAG) é uma condição dermatológica de origem genética com perda progressiva dos cabelos. É o resultado de uma alteração no ciclo do cabelo que leva à miniaturização do folículo com conversão de fios terminais em velo, mais finos, curtos e menos pigmentados. É a causa mais comum de alopecia no sexo masculino, podendo também acometer o sexo feminino em menor proporçãoe de uma forma menos característica (Krause \& Foitzik, 2006; Mulinari-Brenner, Seidel \& Hepp, 2011; Perera, Yip \& Sinclair, 2015).

A AAG possui pico de incidência nos homens após os 50 anos e as estimativas em relação às mulheres são variadas, entretanto, cerca de $30 \%$ do acometimento ocorre por volta dos 70 anos. Sinais precoces de calvície podem também acometer meninos entre 15 e 17 anos (Krause \& Foitzik, 2006; Mulinari-Brenner et al., 2011; Mulinari-Brenner \& Soares, 2009).

A fisiopatogenia é principalmente androgênica, dependente e modulada pelo metabólito da testosterona, adihidrotestosterona (DHT) e pela expressão do folículo piloso, receptor dos andrógenos. ADHT é responsável pela miniaturização dos folículos e desenvolvimento da AAG. Os fatores genéticos também têm sido implicados na patogênese da AAG (Bansal, Manchanda \& Pandey, 2012).

Os indivíduos com AAG podem ter a autoestima afetada, alterando os fatores emocionais com comprometimento da qualidade de vida (Perera et al., 2015; Bansal et al., 2012). O conceito de qualidade de vida é abrangente e envolve além de aspectos objetivos, aspectos subjetivos como preocupações e também o estado emocional do indivíduo. É influenciado por aspectos universais, como bem-estar físico e psicológico, mas também é influenciado culturas pessoais (Totoro, Sousa, Martins, Chacur \& Souza, 2017; Viana \& Junior, 2017). Portanto, o tratamento desta condição é imprescindível, não só para melhorar a condição física, mas também como forma de contribuir na qualidade de vida com aumento da autoestima.

Atualmente, apenas a finasterida oral e o minoxidil tópico são aprovados para tratamento da AAG. Recentemente, alguns estudos mostraram que a cafeína possui efeitos benéficos em pacientes acometidos pela $A A G$ e que um requisito essencial para seu tratamento é a disponibilidade do fármaco via folicular (Bansal et al., 2012; Fischer, Hipler \& Elsner, 2007).

O desenvolvimento de novos produtos é uma importante estratégia para garantir a permanência das empresas no mercado e até a conquista de novos nichos. Portanto, a etapa de pré-formação é uma das atividades fundamentais que antecedem todo o processo para a produção de um cosmético, antes de ser colocado no mercado (Clepf, Martinelli \& Campos, 
2015). Nesse sentido, o objetivo foi realizar um estudo de pré-formulação de shampoo contendo cafeína para tratamento da alopecia androgenética.

\section{MATERIAIS E MÉTODOS}

Na primeira etapa foram selecionados os componentes da formulação do shampoo base para a veiculação dos ativos. A Tabela 1 apresenta as matérias-primas selecionadas e suas respectivas concentrações. Já na Tabela 2 é apresentada a formulação contendo o ativo cafeína. Posteriormente é descrita a técnica de preparo das duas formulações bem como a função e ação terapêutica de cada um dos componentes elegíveis. A manipulação foi realizada em uma farmácia de manipulação conveniada com a Faculdade Patos de Minas. Posteriormente a formulação escolhida foi submetida à avaliação da estabilidade físicoquímica e qualidade visual.

\begin{tabular}{ll}
\hline MATÉRIA PRIMA & $\%$ \\
\hline Lauriléter sulfato sódio & $15 \%$ \\
Lauril éter sulfosuccinatosodio & $23 \%$ \\
Cocoamidopropilbetaína & $5 \%$ \\
Ácido etilenodiaminotetracético & $0,1 \%$ \\
Metilisotiazolinona e fenoxietanol (blend) & $0,5 \%$ \\
Poliquaternio 10 & $0,5 \%$ \\
Decilpoliglucosideo & $5 \%$ \\
Dietanolamina ácido graxo coco & $3 \%$ \\
Água & $9 s p 100 \%$ \\
\hline
\end{tabular}

Tabela 1 - Formulação proposta de Shampoo base

qsp: quantidade suficiente para

\section{MATÉRIA PRIMA}

$\%$

Cafeína

$0,005 \%$

Água destilada

qs

Rev. Psicol Saúde e Debate. Fev., 2018:4(1): 147-160. 
Essência

Shampoo base qs

qsp

Tabela 2 - Formulação do shampoo de cafeína

qs: quantidade suficiente; qsp quantidade suficiente para

\subsection{Técnica de preparo}

Todas as matérias-primas foram pesadas e, primeiramente, em um cálice foram adicionados e homogeneizados o lauril éter sulfato sódio e o lauril éter sulfosuccinato sódio com um pouco de água. Separadamente em um béquer foram adicionados a coco amido propilbetaína, o ácido etilenodiaminotetracético e o blend de metilisotiazolinona e fenoxietanol, posteriormente dissolvidos e homogeneizados em um pouco de água e incorporados ao cálice anterior. Acrescentou-se ao cálice o poliquatérnio 10 e o decilpoliglicosídeo e procedeu-se a homogeneização. A dietanolamina de ácido graxo de coco foi dissolvida separadamente com um pouco de água e incorporada à base. $\mathrm{O}$ pH foi corrigido para 6,5 com ácido cítrico. Completou-se o volume desejado usando água destilada.

Em um béquer, a cafeína foi aquecida em uma chapa com um pouco de água até a completa solubilização. Após o resfriamento, a cafeína foi incorporada ao shampoo base e adicionada a essência e o conservante. Aferiu-se o pH, que deveria estar entre a faixa de $5 \mathrm{a}$ 7.

\subsection{Função dos componentes das formulações}

Lauril éter sulfato sódio

O lauril éter sulfato de sódio é um tensoativo aniônico da classe dos alquil éter sulfatos. São produzidos a partir da etoxilação do alquil sulfato, como o lauril sulfato de sódio, apresentando carga negativa quando solubilizado em água. Apresenta alta solubilidade, sendo utilizado como detergente principal ou primário em formulações de shampoo, apresentando excelente poder de detergência e espuma, além de possuir um baixo custo (Corrêa, 2012). Apresenta boa reserva de viscosidade e sua concentração usual varia de 8 a $12 \%$ de matéria ativa (Ferreira, 2008). 
O lauril éter sulfosuccinato de sódio é um tensoativo aniônico. Forma menos espuma que os tensoativos aquil sulfato e aquil éter sulfato, porém quando utilizado em associação resulta em shampoos com bom poder espumógeno e baixo poder de irritação aos olhos. Dependendo da formulação pode ajudar a manter a viscosidade, possuindo alguma ação condicionadora que melhora a penteabilidade dos cabelos. A concentração usual varia de 18 a 25\%, dependendo do tipo de aplicação (Ferreira, 2008; Barel \& Maibach, 2009).

\section{Cocoamidopropilbetaína}

A cocoamidopropilbetaína é um tensoativo anfotéro, que forma íons negativos ou positivos dependendo do $\mathrm{pH}$ do meio em que estão solubilizados, adquirindo carga positiva em pH ácido ou negativa em pH básico. Apresenta alta compatibilidade com a pele e mucosas devido às suas características suaves e irritabilidade baixa, possuindo alta capacidade de estabilizar a espuma e conferir bom condicionamento. Sua concentração usual varia de 3 a $6 \%$, e quando associada aos alquilsulfatos e aos alquil éter sulfatos, conferem viscosidade ao produto final (Ferreira, 2008; Gomes, 2011).

É importante que a cocoamidopropilbetaína seja utilizada em combinação ao lauril éter sulfato de sódio, pois sozinhos podem favorecer a taxa de irritação, e em associação reduzem essa taxa (Gomes, 2011).

\section{Ácido etilenodiaminatetracético (EDTA)}

Agente sequestrante ou quelante de metais com a função de estabilizar a cor e o odor. Também é capaz de aumentar a eficácia do sistema conservante, pois remove cátions necessários à atividade microbiana. Sua concentração usual varia de 0,05- 0.15\%(Ferreira, 2008). É também utilizado como antioxidante, pois tornam os metais presentes no sistema, indisponíveis para catalisar reações de oxidação (Gomes, 2011).

\section{Metilisotiazolinona e fenoxietanol}

É um blend de conservantes com efeito de sinergismo na ação contra bactérias gram positiva e negativa, fungos e leveduras. Possui fenoxietanol e metilisotiazolinona com propilenoglicol, sendo uma alternativa eficaz aos parabenose liberadores de formaldeído. 
Excelente estabilidade em ampla faixa de pH (2 a 10). Sua concentração usual é de 0,3 a 0,6\% (The Dow Chemical Company, 2017). A concentração máxima de metilisotiazolinona permitida em cosméticos é 0,01\%(Castanedo-Tardana \& Zug, 2013). O Fenoxietanol é considerado seguro para uso em cosméticos até 1\% (Andersen, 2011). O conservante deve ser o último componente a ser acrescentado na formulação e sempre em temperaturas abaixo de $45^{\circ} \mathrm{C}$. O blend de fenoxietanol e metilisotiazolinona pode suportar temperaturas mais altas $\left(70^{\circ} \mathrm{C}\right)$, entretanto a estabilidade na formulação necessita ser confirmada (The Dow Chemical Company, 2017).

\section{Poliquatérnio 10}

O poliquatérnio 10 é um polímero solúvel em água que forma filmes claros e melhora a aparência do cabelo devido à formação de tais filmes. É sintetizado a partir da reação de um sal polimérico quaternário de amônio de hidroxietil celulose com trimetilamônio epóxi substituído. Possui a capacidade de amaciar a superfície do cabelo e melhorar a penteabilidade. Além disso, pode melhorar a textura dos cabelos danificados e malheabilidade. Sua concentração usual pode variar de 0,1 a 1\% (Ferreira, 2008; Gomes, 2011).

\section{Decilpoliglucosídeo}

O decilpoliglucosídeo é um poliglucosídeo graxo formado pela reação de glicose de amido de milho com um álcool graxo natural resultando em um surfactante altamente biodegradável. É não iônico e não contém solventes, caracterizado por não formar carga negativa ou positiva quando em solução aquosa. Baixíssimo potencial de irritação aos olhos e altas propriedades espumógenas. Sua concentração usual é de 4 a 12\% (Ferreira, 2008; Sekhon, 2013).

Dietanolamina de ácido graxo de coco

A dietanolamina de ácido graxo de coco é um tensoativo não iônico, espessante e sobreengordurante. Aumenta a solubilidade do tensoativo primário (lauril éter sulfato de sódio), a formação e estabilização da espuma e melhora a solubilização de essências na formulação. Sua concentração na formulação deve estar entre 10 a $15 \%$ do tensoativos primários (Corrêa, 2012).

Rev. Psicol Saúde e Debate. Fev., 2018:4(1): 147-160. 
Água destilada

A água na formulação do estudo teve a função de veículo da preparação.

\section{Essência}

É um componente opcional da formulação, com função de perfumar. A concentração usual é de 0,2 a 1,0\%, entretanto pode variar em relação à preferência do formulador (Ferreira, 2008).

\section{Cafeína}

A cafeína é um alcalóide psicoestimulante pertencente ao grupo das xantinas, utilizada como estimulante cerebral ou psicomotor. Recentemente, alguns estudos mostraram que a cafeína tem efeitos benéficos em pacientes acometidos pela AAG. O mecanismo proposto que vai contra a miniaturização induzida pelo DHT do folículo capilar inclui a inibição da fosfodiesterase pela cafeína, o que aumenta os níveis de adenosina 3',5'-monofosfato cíclico (AMPc) nas células, promovendo a proliferação e estimulando o metabolismo celular. A enzima fosfodiesterase é responsável pela quebra do AMPc, diminuindo sua concentração nas células e, consequentemente, diminuindo a proliferação capilar. Esta enzima é encontrada naturalmente nos indivíduos e não há correlação com o aumento de sua atividade em pessoas acometidas pela calvície. São os fatores genéticos e/ou hormonais que promovem a apoptose celular e, assim, mais AMPc estaria disponível para a ação da fosfodiesterase (Bansal et al., 2012; Sonthalia, Daulatabad \& Tosti, 2016).

Um estudo in vitro conduzido por Fischer e colaboradores usou amostras de biópsia do couro cabeludo de homens com AAG em que foram usadas diferentes concentrações de testosterona e/ou cafeína por um período de 120 a 192 horas. Na concentração de $0,15 \%$, a cafeína em combinação com testosterona revelou maior supressão nos folículos do que a testosterona sozinha. Entretanto, a cafeína utilizada em concentrações de 0,001\% e 0,005\% teve efeito estimulador, neutralizando os efeitos supressivos da testosterona com alongamento do fio após $120 \mathrm{~h}$ da administração da cafeína em comparação ao grupo controle. Com este estudo foi possível observar que a cafeína possui um papel importante no tratamento da AAG (Fischer et al., 2007).

A via tópica através dos folículos pilosos é a via mais rápida para disponibilização de medicamentos aplicados topicamente. Um requisito essencial para o tratamento da AAG é a 
disponibilidade do fármaco via folicular para a efetividade do tratamento (Otberg et al., 2008). A efetividade da penetração folicular da cafeína aplicada topicamente vem sendo estudada com bons resultados. A penetração folicular da cafeína em uma formulação de shampoo aplicada durante 2 minutos em um estudo, demonstrou que a penetração através dos folículos capilares era mais rápida e maior em comparação com a via interfolicular. Os autoresconcluíram que os folículos pilosos são a únicavia para absorção rápida da cafeína durante os primeiros 20 minutos após a aplicação (Otberg et al., 2008; Otberg et al., 2007).

No estudo de Otberg e colaboradores (Otberg et al., 2008) foi utilizada uma técnica de fechamento seletivo de orifícios foliculares in vivo para a comparação das taxas de absorção folicular e interfolicular da cafeína em seres humanos. Cada único folículo piloso dentro de uma área delimitada da pele foi bloqueado com uma mistura especial de cera. A cafeína em solução foi aplicada topicamente e a absorção transcutânea no sangue foi medida por uma técnica de espectrometria de massa. A cafeína foi detectada $(3,75 \mathrm{ng} \mathrm{ml}(-1))$ em amostras de sangue 5 minutos após a aplicação tópica, quando os folículos permaneceram abertos. Quando os folículos foram bloqueados, a cafeína foi detectável após 20 minutos (2,45 $\mathrm{ng} \mathrm{ml}(-1))$. Os valores mais elevados $(11,75 \mathrm{ng}$ de cafeína $\mathrm{ml}(-1))$ foram encontrados $1 \mathrm{~h}$ após a aplicação, quando os folículos estavam abertos. Com o estudo foi possível demonstrar que os folículos pilosos são pontos fracos consideráveis contra certos medicamentos hidrofílicos e podem permitir uma disponibilidade rápida de substâncias aplicadas topicamente.

Em um ensaio clínico, 40 voluntários usaram uma loção contendo cafeína ao longo de 4 meses. Os resultados dos testes de tração mostraram um aumento da resistência à tração do cabelo e uma diminuição da perda de cabelo em 75\% dos voluntários após 2 meses e em $83 \%$ dos voluntários após 4 meses. O controle dermatológico confirmou a eficácia do produto, com redução importante na perda prematura do cabelo em $43 \%$ dos voluntários, uma melhora na textura (força, resistência à tração) dos cabelos em 53\% deles e uma melhoria das condições do couro cabeludo (eritema, caspa, secura) na maioria dos voluntários que já apresentavam anormalidades no couro cabeludo antes do período do estudo. A avaliação subjetiva da eficácia cosmética mostrou que $80 \%$ dos voluntários ficaram satisfeitos com 0 produto. Em última análise, os autores concluíram que o uso diário da loção é um complemento cosmético eficaz e bem tolerado, sendo a caféina uma substância interessante e promissorano tratamento cosmético da AAG (Bussoletti, Mastropietro, Tolaini \& Celleno, 2011).

Em outro ensaio clínico realizado em 30 voluntários com AAG do sexo masculino foi utilizado shampoo contendo como ativo a cafeína. Os testes foram realizados antes e após 3 
e 6 meses de tratamento com o shampoo. Após três meses de aplicação do shampoo, a progressão da calvície não foi significativamente alterada $(p>0,5)$. No entanto, após 6 meses de aplicação do shampoo a progressão da calvície foi significativamente melhorada $(P=$ 0,004). A extensão da queda do cabelo foi significativamente reduzida após 6 meses de aplicação do shampoo, bem como após 3 meses de aplicação $(p<0,001)$. A avaliação subjetiva da eficácia cosmética mostrou que $67 \%$ dos voluntários estavam satisfeitos com o produto. Particularmente, após 6 meses de tratamento, eles referiram uma diminuição na queda e melhora da força e espessura do cabelo, além de outras condições como prurido e secura (Bussoletti, Mastropietro, Tolaini \& Celleno, 2010).

A cafeína também apresenta efeito esfoliante, estimulando a renovação celular e a circulação sanguínea, colaborando na eliminação de toxinas e células mortas e promovendo uma melhora na saúde dos cabelos (Gomes, 2011).

Suscintamente, os efeitos benéficos da aplicação tópica da cafeína na AAG podem ser atribuídos à inibição da fosfodiesterase, melhora na função de barreira, penetração folicular e estimulação e promoção do crescimento do cabelo. Assim, parece ser um adjuvante útil na gestão da AAG. No entanto, estudos adicionais são necessários para confirmar e estabelecer o papel da cafeína no manejo da AAG (Bansal et al., 2012).

\subsection{Análises dos parâmetros físico-químicos}

Os ensaios físico-químicos asseguram para o consumidor um produto de qualidade. Através deles é possível verificar parâmetros como aspecto, cor, odor, $\mathrm{pH}$, temperatura, densidade, entre outros, que são indicadores de qualidade de cosméticos. Estes são testes simples e rápidos, porém com grande significância (Isaac et al., 2008).

Os ensaios de qualidade foram realizados semanalmente no tempo 0,7 e 15 dias. As amostras foram mantidas em geladeira - GE $\left(5 \pm 2^{\circ} \mathrm{C}\right)$, estufa - ES $\left(40 \pm 2^{\circ} \mathrm{C}\right)$ e em temperatura ambiente - TA (entre $20 \pm 2^{\circ} \mathrm{C}$ ).

\section{Aspecto}

aspecto deve ser analisado para verificação de sinais macroscópicos de instabilidade. A ocorrência de separação de fases, de precipitação, de turvação, é indicativa de instabilidade da amostra. A amostra pode ser descrita como normal, sem alteração; levemente separada, precipitada, turva; separada, precipitada, turva (Isaac et al., 2008) 
Cor

A análise de cor é feita pela comparação visual, sob condições de luz branca. A comparação visual da cor da amostra ensaiada com a cor do padrão foi realizada com cerca de $5 \mathrm{~g}$ da amostra acondicionadas em frascos iguais. A fonte de luz empregada foi a luz branca natural. A amostra pode ser classificada em relação à cor em: normal, sem alteração; levemente modificada; modificada; intensamente modificada (Isaac et al., 2008).

Odor

Também foi realizada pela comparação ao odor do padrão, através do olfato. A amostra pode ser classificada em normal, sem alteração; levemente modificado; modificado; intensamente modificado (Isaac et al., 2008; Brasil, 2010).

\section{Viscosidade}

Para a determinação da viscosidade foi utilizado o Viscosímetro de Efluxo - Modelo tipo Ford e o ensaio realizado em triplicata. A viscosidade será a média dos valores obtidos, expressa em mm2/s ou Centistokes (cSt), sendo permitido um desvio padrão máximo 3\% (Brasil, 2010).

$p H$

A determinação do $\mathrm{pH}$ na amostra foi realizada na amostra a $25^{\circ} \mathrm{C}$, no pHmetro digital, modelo PG 2000, Gehaka previamente calibrado, sem diluições. A primeira determinação fornece valor variável, havendo necessidade de proceder novas leituras. Os valores encontrados posteriormente não deverão variar mais doque 0,05 de unidade em três leituras sucessivas (Brasil, 2010). O resultado correspondeu à média de três determinações.

\section{Densidade}

A análise de densidade foi feita através de balança digital e picnômetro de vidro de $25 \mathrm{~mL}$ em temperatura a 20ㅡ conforme Farmacopéia Brasileira (Brasil, 2010).O resultado correspondeu à média de três determinações. 


\section{RESULTADOS E DISCUSSÃO}

A formulação final apresentou características organolépticas adequadas, e então foi a escolhida para avaliação da estabilidade físico-química e qualidade visual por meio de testes de estabilidade preliminar. A formulação foi colocada em frascos de vidro neutro, transparente, com tampa de boa vedação a fim de evitar perda de gases ou vapor para o meio. As Tabelas 3, 4 e 5 mostram os resultados da avaliação da estabilidade preliminar em geladeira, estufa e temperatura ambiente respectivamente.

\begin{tabular}{lccc}
\hline \multicolumn{1}{c}{ PARÃMETROS } & \multicolumn{3}{c}{ Resultados } \\
\cline { 2 - 4 } & T0 & T7 & T15 \\
\hline Aspecto & sem alteração & sem alteração & sem alteração \\
Cor & sem alteração & sem alteração & sem alteração \\
Odor & sem alteração & sem alteração & sem alteração \\
Viscosidade & $11386 \mathrm{mPa} . \mathrm{s}$ & $7211,1 \mathrm{mPa} . \mathrm{s}$ & $7042 \mathrm{mPa} . \mathrm{s}$ \\
pH & 6,28 & 6,40 & 6,83 \\
Densidade & $1,024 \mathrm{~g} / \mathrm{mL}$ & $1,018 \mathrm{~g} / \mathrm{mL}$ & $1,017 \mathrm{~g} / \mathrm{mL}$ \\
\hline
\end{tabular}

Tabela 3 - Resultados dos parâmetros físico-químicos analisados em geladeira $\left(5 \pm 2^{\circ} \mathrm{C}\right)$.

\begin{tabular}{lccc}
\hline \multicolumn{1}{c}{ PARÂMETROS } & \multicolumn{3}{c}{ Resultados } \\
\cline { 2 - 4 } & T0 & T7 & T15 \\
\hline Aspecto & sem alteração & sem alteração & sem alteração \\
Cor & sem alteração & sem alteração & sem alteração \\
Odor & sem alteração & sem alteração & sem alteração \\
Viscosidade & $11386 \mathrm{mPa} . \mathrm{s}$ & $5697,1 \mathrm{mPa} . \mathrm{s}$ & $5512,9 \mathrm{mPa} . \mathrm{s}$ \\
pH & 6,28 & 7,0 & 6,89 \\
Densidade & $1,024 \mathrm{~g} / \mathrm{mL}$ & $1,01 \mathrm{~g} / \mathrm{mL}$ & $1,009 \mathrm{~g} / \mathrm{mL}$ \\
\hline
\end{tabular}

Tabela 4 - Resultados dos parâmetros físico-químicos analisados em estufa $\left(40 \pm 2^{\circ} \mathrm{C}\right)$.

Rev. Psicol Saúde e Debate. Fev., 2018:4(1): 147-160. 


\begin{tabular}{lccc}
\hline \multicolumn{1}{c}{ PARÂMETROS } & \multicolumn{3}{c}{ Resultados } \\
\cline { 2 - 4 } & T0 & T7 & T15 \\
\hline Aspecto & sem alteração & sem alteração & sem alteração \\
Cor & sem alteração & sem alteração & sem alteração \\
Odor & sem alteração & sem alteração & sem alteração \\
Viscosidade & $11386 \mathrm{mPa} . \mathrm{s}$ & $5697,1 \mathrm{mPa} . \mathrm{s}$ & $5512,9 \mathrm{mPa} . \mathrm{s}$ \\
pH & 6,28 & 6,41 & 6,49 \\
Densidade & $1,024 \mathrm{~g} / \mathrm{mL}$ & $1,033 \mathrm{~g} / \mathrm{mL}$ & $1,037 \mathrm{~g} / \mathrm{mL}$
\end{tabular}

Tabela 5 - Resultados dos parâmetros físico-químicos analisados em temperatura ambiente $(20 \pm$ $\left.2^{\circ} \mathrm{C}\right)$.

No desenvolvimento de cosméticos, o teste de estabilidade preliminar é considerado um teste orientativo, podendo ser realizado em um curto intervalo de tempo. O teste consiste em submeter a amostra a condições extremas de temperatura, acelerando assim, os processos de instabilidade. Tem como objetivo auxiliar na triagem e orientar a escolha das formulações (Isaac et al., 2008; Brasil, 2004).

Quanto às características organolépticas não houve nenhuma alteração na coloração, homogeneidade e odor das amostras submetidas às condições extremas de temperatura durante todo o tempo do estudo.

Essas avaliações são fundamentais para determinar a qualidade da formulação, pois quaisquer alterações nessas características podem ser um indicativo de alterações químicas ou contaminações microbiológicas (Lourenço \& Lyra, 2015).

Sendo assim, após os resultados do teste preliminar foi possível direcionar a escolha da formulação que se manteve estável até a última análise para a continuidade de estudos de estabilidade, eficácia e aceitabilidade.

\section{CONSIDERAÇÕES FINAIS}

A formulação atendeu aos critérios de aceitação definidos, apresentando boa aparência e consistência, além de aspecto homogêneo. A formulação proposta é viável ao universo magistral e, em termos de análises físico-químicas, não apresentou qualquer instabilidade, mantendo-se estável até a última análise. A formulação poderá ser a escolha 
para a continuidade de estudos de estabilidade para determinação do prazo de validade ideal, eficácia e aceitabilidade.

\section{REFERÊNCIAS}

Andersen, FA (2011). Annual Review of Cosmetic Ingredient Safety Assessments: 2007-2011. International Journal of Toxicology. 30(5), 73-127.

Bansal, M., Manchanda, K., Pandey, S.S (2012). Role of Caffeine in the Management of Androgenetic Alopecia. Int. J. Trichology. 4(1), 185-186.

Barel, A.O., Maibach, H.I.(2009). Handbook of Cosmetic Science and Technology. New York: Informa Health care Publishers; 5ed.

Brasil (2004). Guia de Estabilidade de Produtos Cosméticos. Brasília: Anvisa. Recuperado em 11 de novembro, 2017, de http://www.crq4.org.br/downloads/guia_cosme.pdf.

Brasil (2010). Farmacopeia Brasileira. Brasília: Agência Nacional de Vigilância Sanitária; 5ed.

Bussoletti, C., Mastropietro, F., Tolaini, MV., Celleno, L (2010). Use of a Caffeine shampoo for the treatment of male androgenetic alopecia. J. Appl. Cosmetol. 28(4), 153-162.

Bussoletti, C., Mastropietro, F., Tolaini, MV., Celleno, L (2011). Use of a cosmetic caffeine lotion in the treatment of male androgenetic alopecia. J. Appl. Cosmetol. 29(1), 167-168.

Castanedo-Tardana, M.P., \& Zug, K.A (2013). Methylisothiazolinone. Dermatitis. 24(1), 2-6.

Clepf, S., Martinelli, D. P. C., \& Campos, P. M. B. G. M (2015). Visão sistêmica no desenvolvimento de produtos cosméticos. CPMark - Caderno Profissional de Marketing. 3(4), 329-337.

Corrêa, M.A (2012). Cosmetologia ciência e técnica. São Paulo: Livraria e Editora Medfarma.

Ferreira, A. (2008). Guia prático de farmácia magistral. São Paulo: Pharmabooks; 5ed.

Fischer, T.W., Hipler, U.C., Elsner, P (2007). Effect of caffeine and testosterone on the proliferation of human hair follicles in vitro. Int. J. Dermatol. 46(1), 27-35.

Gomes JGCC (2011). Estudos de pré-formulação e desenvolvimento de preparações cosméticas. Araraquara: Universidade Estadual Paulista. Recuperado em 25 novembro, 2016, de

https://repositorio.unesp.br/bitstream/handle/11449/119287/gomes_jgcc_tcc_arafcf.pdf?sequ ence $=1$ \& is Allowed $=\mathrm{y}$.

Issac, V.L.B., Cefali, L.C., Chiari, B.G., Oliveira, C.C.L.G., Salgado, H.R.N., \& Correa, M.A (2008). Protocolo para ensaios físico-químocos de estabilidade de fitocosméticos. Cienc. Farm. Básica, 29(1), 81-96. 
Krause, K., \& Foitzik, K (2006). Biology of the hair follicle: the basics. Semin. Cutan. Med. Surg. 25(1), 2-10.

Lourenço, E.A.D., \& Lyra M.A.M.M (2015). Desenvolvimento e estudo de estabilidade de xampu anti-caspa a base de piritionato de zinco 2\%. Revista Eletrônica Estácio Recife. 1(1), $1-7$.

Mulinari-Brenner, F., Seidel G., Hepp T (2011). Entendendo a alopecia androgenética. Surg. Cosmet. Dermatol. 3(4), 329-337.

Mulinari-Brenner F., \& Soares IF (2009). Alopecia androgenética masculina: uma atualização. Rev. Ciênc. Méd. 18(3), 153-161.

Otberg, N., Patzelt, A., Rasulev, U., Hagemeister, T., Linscheid, M., Sinkgraven, R., Sterry, W., \& Lademann, J (2008). The role of hair follicles in the percutaneous absorption of caffeine. Br. J. Clin. Pharmacol. 65(4), 488-492.

Otberg, N., Teichmann, A., Rasuljev, U., Sinkgraven, R., Sterry, W., \& Lademann, J (2007). Follicular penetration of topically applied caffeine via a shampoo formulation. Skin. Pharmacol. Physiol. 20(4), 195-8.

Perera, E., Yip, L., \& Sinclair, R.(2015). Alopecia Areata. Hair Ther Transplant. 47, 67-75.

Sekhon, B.S (2013). Surfactants: Pharmaceutical and medicinal aspects. J. Pharm. Technol. 1, 11-36.

Sonthalia, S., Daulatabad, D., Tosti, A (2016). Hair Restoration in Androgenetic Alopecia: Looking Beyond Minoxidil, Finaste ride and Hair Transplantation. J. Cosmo. Trichol. 2(1), 1000105.

The Dow Chemical Company. (2017). Neolone PE. Recuperado em 11 dezembro, 2016, de http://msdssearch.dow.com/PublishedLiteratureDOWCOM/dh 0972/0901b803809725b3.pdf ?filepath=microbial/pdfs/noreg/253-02870.pdf\&fromPage=GetDoc.

Totoro, R., Sousa, M., Martins, R., Chacur, E., \& Souza, F (2017). Avaliação da qualidade de vida dos usuários de um núcleo de apoio à saúde da família - Nasf. Psicologia e Saúde em Debate. 3,50-67.

Viana, A., Junior, G.A (2017). Qualidade de vida em idosos praticantes de atividades físicas. Psicologia e Saúde em Debate, 3(1), 87-98. 